\title{
Idiopathic Recurrent Meningitis-A Rare Presentation of Congenital Peri lymphatic Fistula
}

\author{
Anand R*, Sankya Shanmugam and Minu Madeswaran \\ Department of Otolaryngology, Anand ENT Hospitals Coimbatore, India \\ *Corresponding author: Anand R, Department of ENT, Anand ENT Hospitals Coimbatore, India
}

\begin{abstract}
Perilymph fistula is caused by an abnormal communication between the perilymph space and the middle ear. It can either be congenital or acquired. In patients presenting with idiopathic bacterial meningitis, we as otolaryngologists should have a high index of suspicion towards peri lymphatic fistula. Nowadays, with screening of newborn with Computed Tomography temporal bone, inner ear malformations have been diagnosed more often without any signs of meningitis. We here present a case of congenital perilymph fistula in a pediatric patient with recurrent episodes of idiopathic meningitis. A brief review of the literature on this entity confirms the difficulty of making a definitive diagnosis in most cases.
\end{abstract}

Keywords: Perilymph fistula; idiopathic recurrent meningitis; CSF leak

Abbreviations: CSF: Cerebrospinal Fluid; PLF: Peri Lymphatic Fistula; HRCT: High Resolution Computed Tomography

\section{Introduction}

Congenital Peri lymphatic fistula are spontaneous leaks of perilymph (cerebrospinal fluid) into the middle ear as a result of a congenital weakness or defect in the temporal bone with no previous history of trauma and exertion. Recurrent meningitis secondary to congenital labyrinthine anomaly is relatively a rare clinical entity and its diagnosis depends on clinical, radiological and intra operative features.

\section{Case Report}

We present a case of recurrent meningitis secondary to congenital peri lymphatic fistula. 7-year-old boy was referred from pediatric department to us to rule out any ENT foci for recurrent Idiopathic meningitis.

Patient suffered from four episodes of meningitis in the past and had about every six months and he recovered completely after each episode. He underwent all the necessary investigations which was found to be normal. MR imaging of brain was normal. Lumbar puncture showed picture of meningitis , but foci of infection could not be found. He was referred to us when he gave a peculiar history URI and earache which preceded an episode of meningitis. Initially it was suspected that the foci of infection were from tonsils, for which he underwent adenotonsillectomy, despite that he again developed meningitis with complaints of ear fullness and hearing loss. Otoendoscopic findings in the right ear showed clear fluid behind intact tympanic membrane and left ear was found to be normal. Audiometry showed moderate to severe sensorineural hearing loss \& Tympanometry showed B type tympanogram in the right ear. Since fluid was in the middle ear, Perilymph fistula was suspected. Myringotomy was performed. Clear fluid from the middle ear was sent for CSF analysis and was confirmed to be the same. Computed tomography of temporal bone showed defect at the junction of footplate of stapes and promontory (Figures 1-3). Tympanmastoid exploration was performed. Intraoperative findings showed clear fluid between footplate junction and facial canal. Head of malleus and incus were removed and examined for any defect in areas of attic and eustachian tube .The site of leak was confirmed and was covered with fat, temporalis muscle and temporalis fascia (Figures 4-7). Patient was in regular follow up for next 1 year and there were no further episodes of meningitis . 


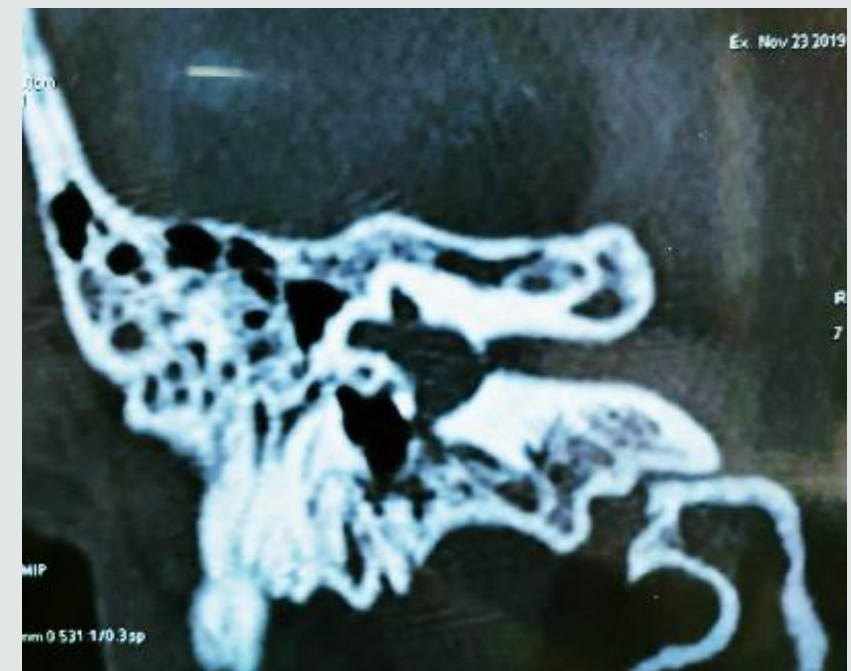

Figure 1.

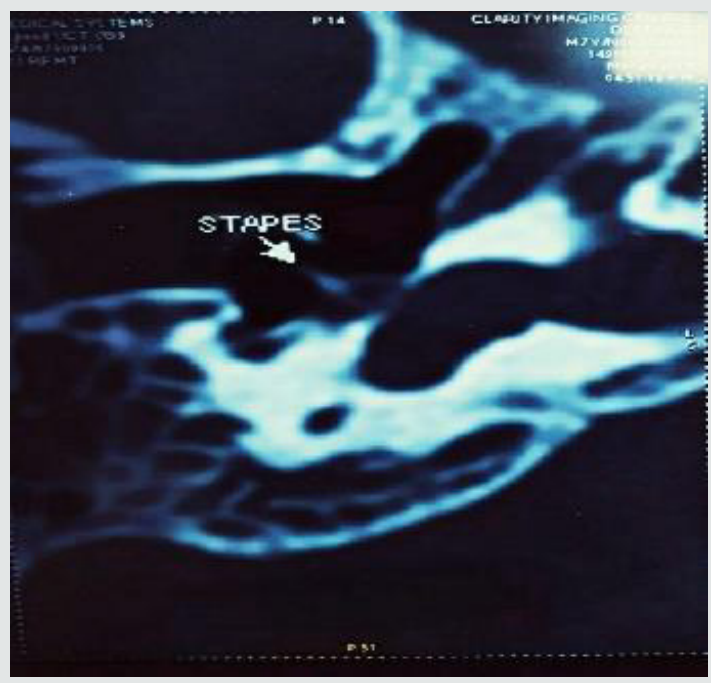

Figure 2.

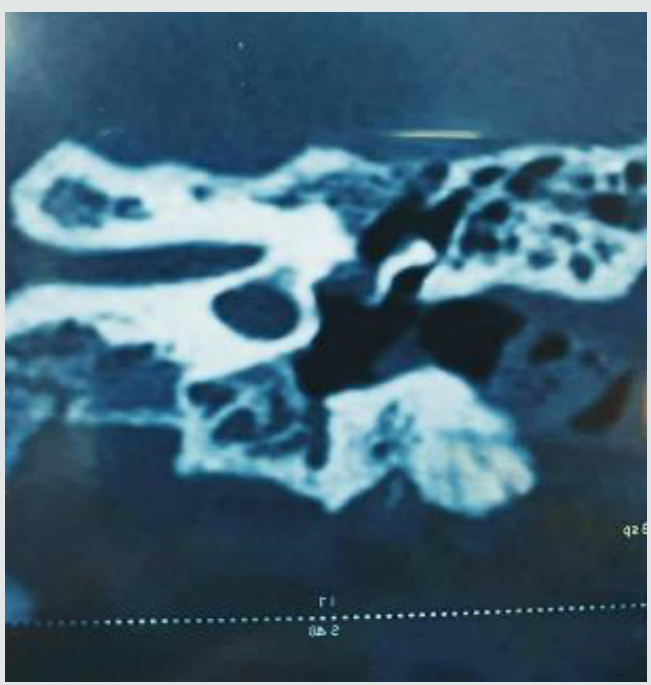

Figure 3.

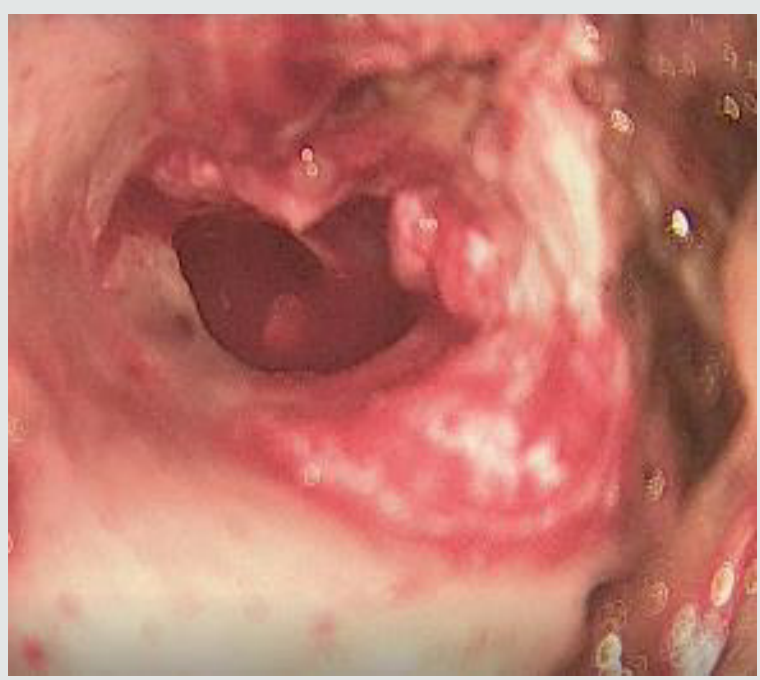

Figure 4 .

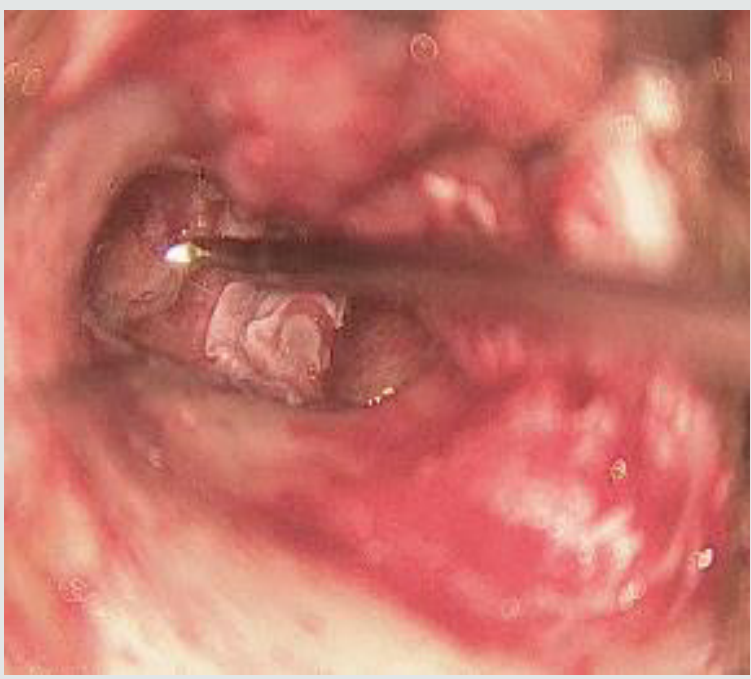

Figure 5.

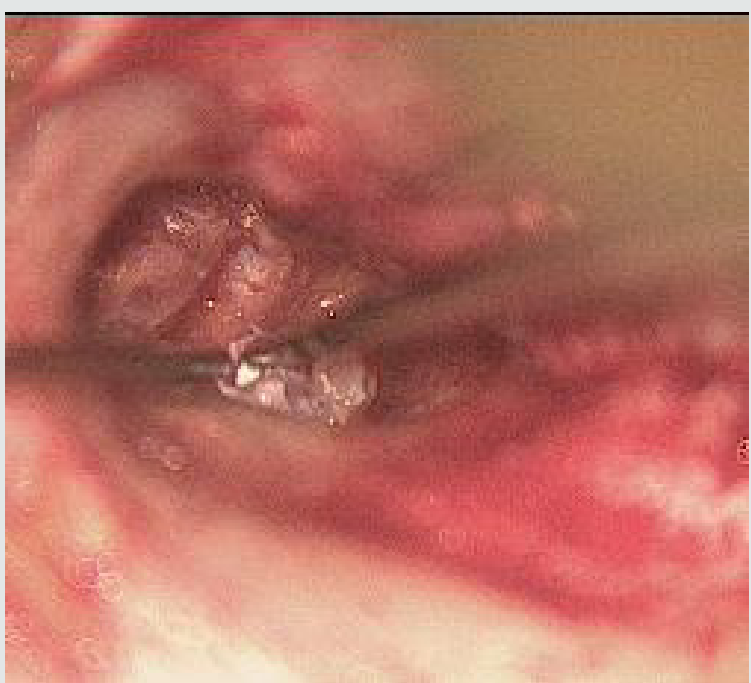

Figure 6. 


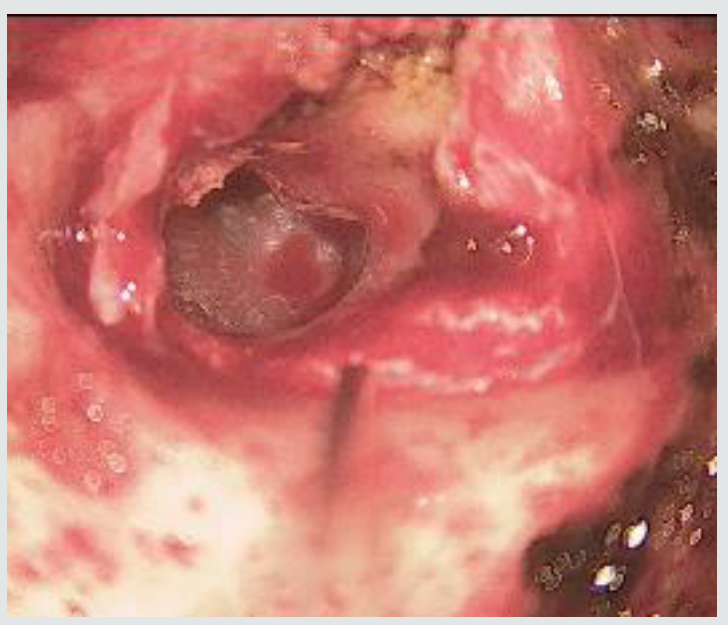

Figure 7.

\section{Discussion}

Bacterial meningitis in childhood is a life-threatening infection of the central nervous system that is mostly associated with inner ear malformations and Cerebrospinal Fluid (CSF) leaks. Up to $33 \%$ of cases of repeated meningitis in children are caused by otolaryngologic etiologies [1] Congenital peri lymphatic fistula (PLF) is an abnormal communication between the middle and inner ear. It may be associated with micro fissures around the oval and round windows or labyrinthine (IAM dysplasia) [2]. The basic abnormality is a fistulous communication between the inner ear and the intracranial compartment as well as another communication between the inner ear and middle ear. In some cases, as in Hyrtl's fissure, there is a direct communication between the subarachnoid space of the posterior cranial fossa and the middle ear [3].

Developmental anomaly: The otic placodes develop from the ectoderm at the third week of intrauterine life and invaginate to form otic vesicles. These subsequently develop to form the endolymphatic sac, cochlea, and vestibule by the fifth week. The membranous cochlea develops 1.5 turns at the end of 6 weeks and 2.5 turns by the end of the seventh week. The semicircular canals develop by the eighth week. The development of the inner ear is almost complete by the eighth week, so any insult during this period can produce several anomalies including congenital perilymph fistula [3].

Diagnosis of PLF, however, is quite difficult and delayed because of low index of suspicion and the main challenge is the identification of those patients that need to undergo an exploratory tympanotomy [4], there are no clinical-audiologic symptoms or radiographic indicators that can be considered pathognomonic of perilymph fistula. Hearing loss may be there which can be either fluctuating, progressive, or sudden. It can be associated with vertigo; patient may present with meningitis Establishing a causal relationship between the inner ear and repeated episodes of meningitis involves detailed audiologic and radiologic investigations [2]. Testing for beta-2 transferrin may yield results, but the only true way to diagnose for PLF is intraoperatively [4]. Choice of Imaging Modality is High Resolution Computed Tomography of Temporal bone Bony delineation is well defined with HRCT and thin slice MRI of temporal bone can highlight fluid filled communications [57]. The most frequent middle ear anomalies identified in children with congenital PLF are a deformity of the stapes and lateral-facing round window [8]. Certain type of malformation of the inner ear, with spontaneous peri lymphatic fistula is seen

a) An internal auditory meatus in the form of a funnel, in which the narrow part corresponds to the fundus of the internal auditory meatus.

b) A generalized dilatation of the osseous labyrinth, different from Mondini's malformation, in which only the basal turn of the cochlea, the aqueduct of the vestibule and the lateral semicircular canal are affected [9].

Inner ear malformations may involve bone defects of the stapes footplate. Rao et al. reported that $59 \%$ of cases of repeated bacterial meningitis in children were due to anatomical congenital malformations of the temporal bone [10]. Savva et al. [4] reported that persistent CSF leaks from inner ear malformations were observed in $15 \%$ to $25 \%$ of repeated bacterial meningitis cases in children [11]. Surgical exploration is required in case of recurrent meningitis. Obliteration of the fistula with temporal muscle and fascia has prevented the recurrence of meningitis. the middle ear exploration is considered to be less difficult and more reliable than the intracranial approach. In repairing the CSF leak, many different materials and tissues have been used, including temporal fascia, temporalis muscle, siliconized rubber, absorbable gelatin film, and acrylic compounds [12,13].

\section{Conclusion}

The diagnosis of perilymph fistula is controversial. The variability among patients makes perilymph fistula difficult to diagnose. High index of suspicion should be there for inner ear anomaly if a patient presents with recurrent episodes of idiopathic meningitis. Undiagnosed cases will have high mortality rate. Paediatric congenital perilymph fistula will not have classical symptoms as in adults hence early diagnosis and management is important.

\section{References}

1. Rupa V, Agarwal I, Rajshekhar V (2014) Congenital perilymph fistula causing recurrent meningitis: lessons learnt from a single-institution case series. Otolaryngol Head Neck Surg 150(2): 285-291.

2. Jonathan P Harcourt (2018) Vestibular Rehabilitation. Scott Brown's Textbook of Otolaryngology-Audit audiology. $8^{\text {th }}$ Edn 1: 111-112.

3. Rupa V, Rajshekhar V, Weider DJ (2000) Syndrome of recurrent meningitis due to congenital perilymph fistula with two different clinical presentations. Int J Pediatr Otorhinolaryngol 54(2-3): 173-177. 
4. Weber PC, Kelly RH, Bluestone CD (1994) Beta-2 transferrin confirms perilymphatic fistula in children. Otolaryngol Head Neck Surg 110(4): 381-386.

5. Bluestone CD, Klein JO (2001) Otitis media in Infant and children .Philadelphia Saunders pp. 239-241.

6. Johnson DB, Brennan P, Toland J, O Dwyer AJ (1996) Magnetic resonance imaging in the evaluation of cerebrospinal fluid fistulae. Clin Radiol 51(12): 837-841.

7. Gupta V, Goyal M, Mishra NK, Sharma A, Gaikwad SB (1997) Positional MRI: a technique for confirming the site of leakage in cerebrospinal fluid rhinorrhoea. Neuroradiology 39(11): 818-820.

8. Isono M, Murata K, Aiba K, Miyashita, Weissman JL, Weber PC, Bluestone CD (1994) Congenital perilymphatic fistula: computed tomography appearance of middle ear and inner ear anomalies. OtolaryngologyHead and Neck Surgery 111(3 Pt 1): 243-249.
9. Phelps P, Lloyd GAS (1978) Congenital deformity of the internal auditory meatus and labyrinth associated with cerebrospinal fluid fistula. Adv Oto-Rhino-Laryngol 24: 51-57.

10. Rao AK, Merenda DM, Wetmore SJ (2005) Diagnosis and management of spontaneous CSF otorrhoea. Otol Neurotol 26(6): 1171-1175.

11. Savva A, Taylor MJ, Beatty CW (2003) Management of cerebrospinal fluid leaks involving the temporal bone: report on 92 patients. Laryngoscope 113(1): 50-56.

12. Farrior B, Endicott JN (1971) Congenital mixed deafness: Cerebrospinal fluid otorrhea: Ablation of the aqueduct of the cochlea. Laryngoscope 81(5): 684-699.

13. Bottema T (1975) Spontaneous cerebrospinal fluid otorrhea: Congenital anomaly of bony labyrinth a possible cause. Arch Otolaryngol 101: 693694.

\section{(c) (1) \\ This work is licensed under Creative Commons Attribution 4.0 License}

To Submit Your Article Click Here: Submit Article
DOI: $10.32474 /$ SJO.2020.04.000198

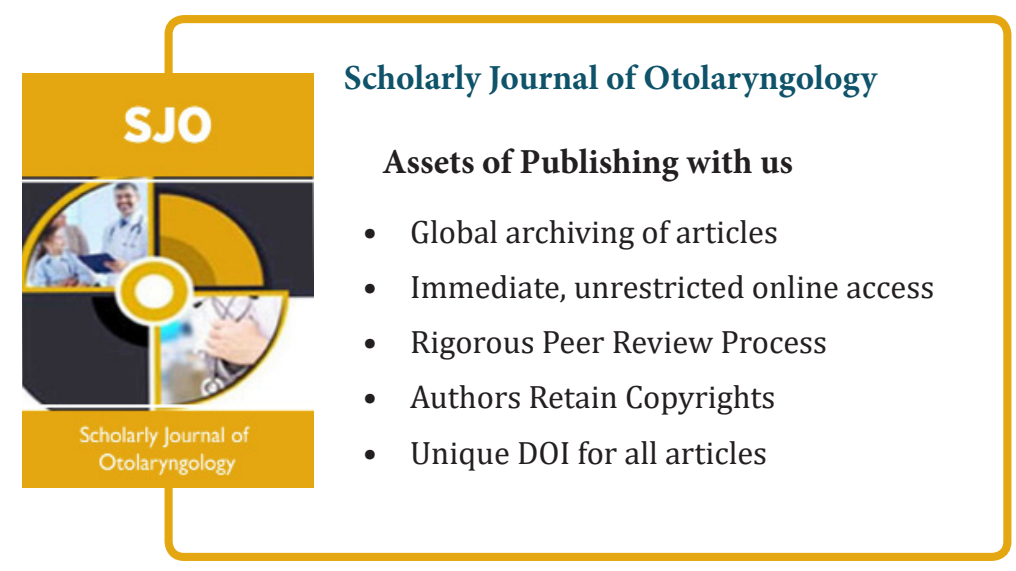

